Mediterránea Ser. Biol. (1990), n. ${ }^{\circ}$ 12. Pág. 97-112

\title{
CICLOS ESTACIONALES DE ACTIVIDAD Y MODELOS ESPACIALES EN LAS POBLACIONES DE COLEOPTERA TENEBRIONIDAE DE LAS DUNAS COSTERAS DE LA PLAYA DE EL SALADAR (ALICANTE-ELCHE, SE. DE ESPAÑA)
}

\author{
por \\ CARLOS MARTÍN CANTARINO Y EDUARDO SEVA ROMÁN (1)
}

\section{RESUMEN}

Se estudian los ciclos de actividad estacional y los modelos de distribución en el espacio de cuatro especies de coleópteros Tenebrionidae (Pimelia modesta Hbst., Erodius carinatus Sol., Tentyria elongata Walt, y Pachychila frioli Sol.) mediante el uso de trampas de caída en un área dunar costera de la zona de Alicante (SE de España). Tres de las especies ( $P$. modesta, $T$. elongata y $P$. frioli) muestran períodos de actividad bastante largos (primavera-otoño), mientras que $E$. carinatus muestra un ciclo de actividad de tipo corto, restringido a la temporada primaveral. Se comparan estos modelos fenológicos con los de otros Tenebrionidae mediterráneos.

El análisis de la distribución espacial se centró en la discriminación de las preferencias de hábitat y su relación con las variaciones temporales de los índices de agregación. P. modesta, $E$. carinatus y $P$. frioli ocupan preferentemente las zonas de arenas no consolidadas, mientras que $P$. elongata parece seleccionar los sustratos semiconsolidados. Se destaca que todas las especies tienden a concentrarse, durante los períodos de máxima agregación, en los microhábitat preferidos. Se discuten asimismo las posibles relaciones existentes entre estas preferencias de microhábitat y las distintas estructuras morfológicas de adaptación al sustrato de los Tenebrionidae.

\section{SUMMARY}

The annual activity cycles and the patterns of spatial distribution of four species of Coleoptera Tenebrionidae: Pimelia modesta Hbst., Erodius carinatus Sol., Tentyria elongata Walt. and Pachychila fioli Sol., were studied by means of pitfall traps at a coastal dune near Alicante (SE Spain). Three species (Pimelia modesta, Tentyria elongata and Pachychila frioli) showed long-

(1) Departamento de Ciencias Ambientales y Recursos Naturales. Facultad de Ciencias. Universidad de Alicante. Apartado 99. Alicante (España). 
termed activity periods, white Erodius carinatus has a very much shorter one. These patterns are compared with the seasonal rhythms of other Mediterranean Tenebrionidae.

The spatial pattern analysis was centred in the discrimination of habitat preferences and its relation with temporal variations in the aggregation indices. Pimelia modesta, Erodius carinatus and Pachychila frioli select preferably the zones os non-consolidated sand, but Tentyria elongata occurs in larger numbers in semi-consolidated substrates. It is remarked that all the species tend to clump, at the periods of maximal aggregation, in the prefered microhabitats. The relationships between microhabitat preferences and the morfological adaptative features of the Tenebrionidae are discussed.

Key words: Coleoptera, Tenebrionidae; pipfall-trapping; spatial distributions; activity cycles; sand-dune ecosystems; Spain.

\section{INTRODUCCIÓN}

Los ciclos de actividad y los modelos de distribución espacial de las poblaciones animales vienen determinados por la forma peculiar en que cada una de ellas responde a la heterogeneidad espacial y temporal de su medio. Son, por tanto, reflejo de las características adaptativas de la especie y su estudio presente un gran interés a la hora de intentar comprender la ecología poblacional de la misma.

En el caso de los artrópodos terrestres de superficie, el muestreo mediante trampas de caída («pitfall traps») se ha revelado como una de las técnicas más fructíferas en este tipo de análisis (DESENDER \& MAELFAIT, 1986). Aunque la interpretación de los datos obtenidos con estos muestreos no deja de plantear problemas ( Vid. ADIS, 1979; SOUTHWOOD, 1966; GREENSLA$\mathrm{DE}, 1964)$, lo cierto es que la gran cantidad de datos proporcionados los hace sumamente valiosos siempre que se controlen distintos factores que puedan distorsionar los resultados (BAARS, 1979).

Las tampas de caída no proporcionan información directa sobre las variaciones en los niveles de densidad de las poblaciones, sino más bien de sus niveles de actividad. Ha habido, no obstante, diversos intentos de relacionar las capturas obtenidas mediante estos métodos con los tamaños poblacionales (BAARS, 1979; DEN BOER, 1979). Igualmente, se ha procurado establecer relaciones entre el número de capturas y diversos parámetros físicos como la temperatura (LUFF, 1982).

Con respecto a la distribución espacial, la mayoría de los trabajos se ha centrado en la discriminación de las preferencias de hábitat (p. ej., CALKINS \& KIRK, 1973; DESENDER \& MAELFAIT, 1980; RICKARD, 1970, etc.). Pocos, por el contrario, han intentado un estudio de los modelos de distribución teniendo en cuenta el estado agregativo de las poblaciones y sus variaciones con el tiempo (GREENSLADE, 1963; LUFF, 1986; DE LOS SANTOS, 1982). Ello contrasta con la importancia que, sin embargo, se ha reconocido a estas pautas de distribución en los estudios de ecología de poblaciones, dada su influencia en la eficiacia del muestreo y en la correcta interpretación biológica de sus resultados (TAYLOR, 1981; DE LOS SANTOS, 1982). 
En el presente trabajo se persiguen dos objetivos fundamentales. Por un lado, proporcionar datos sobre los ciclos de actividad de cuatro especies de Tenebrionidae epigeos de las dunas costeras de El Saladar (Alicante-Elche). Por otro, obtener los modelos de distribución espacial de las mismas, analizando la relación existente entre las variaciones en el estado agregativo y los niveles de ocupación de los diferentes microhábitat. Se pretende así estudiar la influencia que tales estados agregativos tienen sobre los datos del muestreo, a fin de intentar una mejor aproximación al verdadero sentido ecológico de las pautas de ocupación del territorio.

\section{ÁREA DE ESTUDIO Y MÉTODOS}

Hasta hace unas pocas décadas, la zona litoral extendida desde unos $5 \mathrm{ki}$ lómetros al Sur de la ciudad de Alicante, en el SE. de España, hasta las estribaciones septentrionales de la Sierra de Santa Pola, es decir, la mitad merional de la llamada «Bahía de Alicante», constituía un extenso y continuo campo de dunas de unos 10 kilómetros de longitud en sentido N-S. Profundamente afectada por las actividades turísticas que se han venido promoviendo en la zona desde los años 60, en la actualidad buena parte de esta extensión ha sido cubierta por edificios y por vías asfaltadas. Sin embargo, aún se conservan dos áreas en su estado natural, de las cuales la mayor, con unas $100 \mathrm{Ha}$. de superficie, es la que corresponde a la denominada Platja del Saladar, en término municipal de Elche (SEVA, 1982) (figura 1).

La vegetación de esta zona presenta en líneas generales los rasgos típicos del conjunto de los arenales costeros mediterráneos. Así, cabe distinguir varias comunidades vegetales en función del grado de movilidad del sustrato sobre el que se asientan. En las arenas más móviles, correspondientes a la zona más cercana a la línea de costa o a puntos en los que la disturbación producida por el viento u otros factores impide la consolidación de las arenas, se instalan comunidades encuadrables en la alianza fitosociológica Ammophilion arundinaceae $\mathrm{Br}$.-Bl-1933, con Ammophila arenaria (L) Link var. arundinacea (Host) Husnot, Elymus farctus (Viv.) Runemark ex Melderis, Medicago marina L., y Lotus creticus L., como especies localmente más abundantes. Allí donde el grado de fijación de la arena es mayor, aparecen las comunidades de la alianza Crucianellion maritimae Rivas Goday \& Rivas Martínez, 1963, con Crucianella maritima 1., Thymelaea hirsuta (Lamk.) Ceballos-Vicioso, Helichrysum stoechas (L)DC, Teucrium belion Schreber y Ononis natrix L. ssp ramosissima Ball. \& Trabut. No se presentan en la zona comunidades correspondientes a grados mayores de consolidación de la duna, tales como las asociaciones sabulícolas de Rosmarino-Ericion o los matorrales de Juniperion lyciae.

En las dunas de El Saladar, los representantes de la familia Tenebrionidae constituyen uno de los elementos más conspicuos de la entomofauna. SAULEDA (1977) cita en la zona 13 especies de Tenebriónidos psammófilos estrictos. Dentro de esta taxocenosis, existe un grupo de cuatro especies bien diferenciado: Pimelia modesta Hbst., Erodius carinatus Sol., Tentyria elongata Ealt. y Pachychila fioli Sol. Todas ellas presentan actividad estival y diurna y destacan del resto de la tenebrionidofauna por su tamaño comparativamente grande y su coloración negra. La labor de muestreo se centró en estas cuatro 


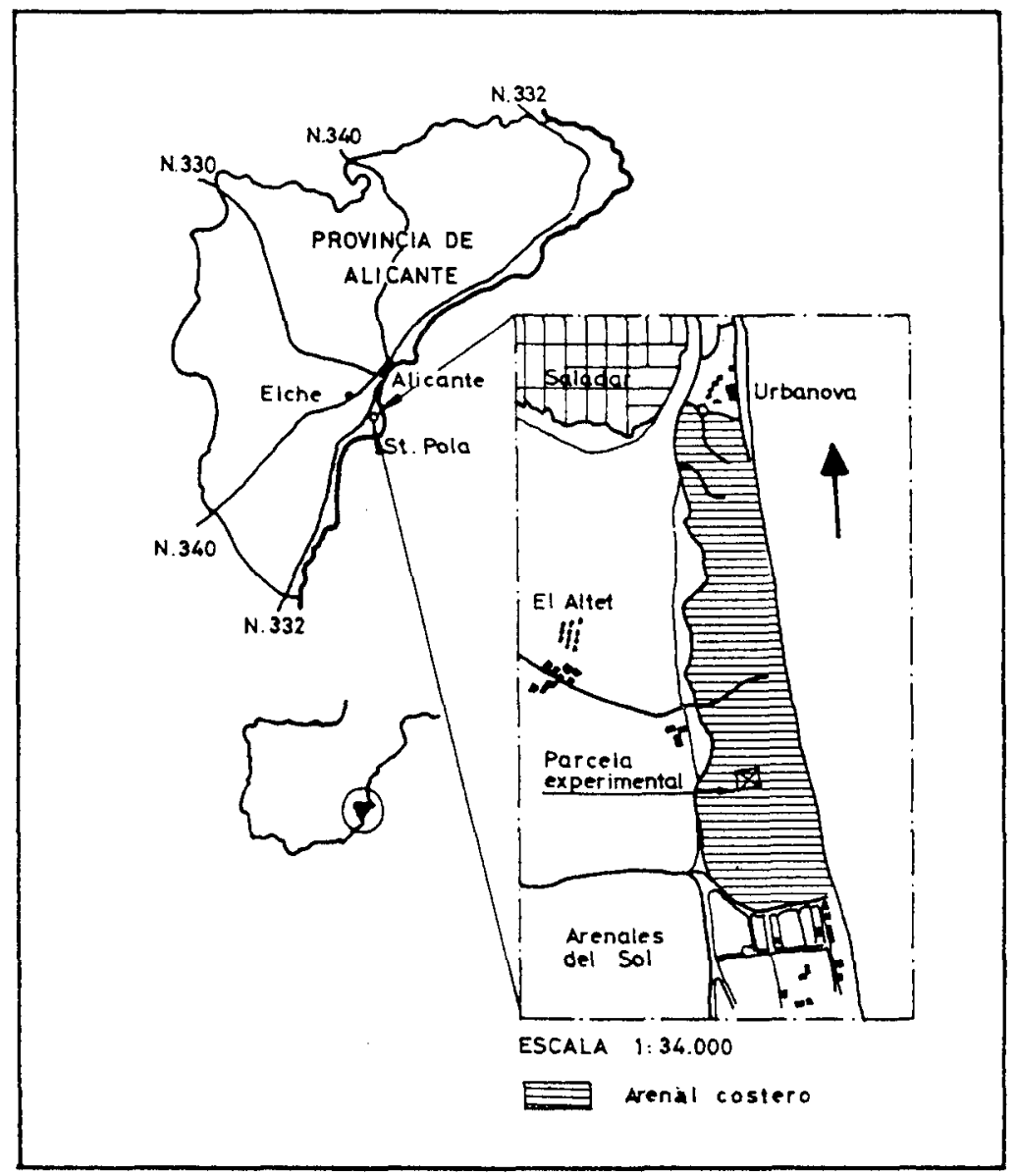

Fig. 1.-Mapa situación.

especies, si bien se recolectó abundante información adicional sobre algunos otros coleópteros epigeos que será objeto de una próxima publicación.

La parcela de estudio se escogió de manera que en la misma se hallaran representados los principales tipos de vegetación psammófila. Ocupa una extensión de unos $3.000 \mathrm{~m}^{2}$ y se encuentra situada a unos $300 \mathrm{~m}$. de la línea de costa. El dispositivo de muestreo consistió en una batería de 20 trampas de caída dispuestas en cuatro filas de cinco trampas cada una, con separación de $10 \mathrm{~m}$. entre las filas y entre las trampas de una misma fila. Las trampas estaban realizadas con recipientes cilíndricos de plástico, de $17 \mathrm{~cm}$. de diámetro de boca y $20 \mathrm{~cm}$. de profundidad. Cada recipiente disponía en su fondo de dos o cuatro orificios cubiertos con rejilla de plástico a fin de permitir el dre- 




Fig. 2.-Plano parcela.

naje de la trampa en caso de lluvia. No se colocó ningún tipo de cebo o conservante.

Los muestreos se realizaron semanalmente durante todo 1988, con recuento y marcaje de los individuos en cada sesión y liberación de los mismos en las cercanías de la tampa.

Dentro de la parcela de nuestreo se delimitaron tres subparcelas, cuya cubierta vegetal presentaba características más o menos uniformes (Figura 2). La zona $\mathrm{A}$, que comprendía cinco trampas, correspondía a un territorio de arenas escasamente fijadas y vegetación tipo Ammophilion puro. La zona B, con seis trampas, mostraba una vegetación de transición entre Ammophilion y Crucianellion, con Crucianella maritima y Lotus creticus como especies dominantes. La zona $\mathrm{C}$, con dos trampas, correspondía al tipo de vegetación de Crucianellion puro y, por tanto, a las arenas de cierto grado de consolidación. El resto de trampas, situadas en zonas cuya vegetación y tipo de sustrato eran complejos y de difícil caracterización, no se tuvo en cuenta para el análisis de las preferencias de hábitat. 


\section{RESULTADOS}

En la figura 3 se muestra el desarrollo de los períodos de actividad de las cuatro especies, tal como viene indicado por el número medio de capturas por trampa en cada muestreo.

Tres de las especies (Pimelia modesta, Tentyria elongata y Pachychila frioli) presentan períodos de actividad extendidos desde el inicio de la primavera hasta finales de verano. Sin embargo, existen claras diferencias interespecíficas por lo que respecta a la situación temporal de los máximos de actividad. Así, Pachychila frioli muestra dos marcados picos, uno en abril-mayo y otro en agosto. Tentyria elongata, por su parte, que presenta un máximo en agosto pararelo al de Pachychila, registra un pico primaveral menos marcado y algo más temprano que el de ésta. Pimelia modesta, tras su aparición en marzo, alcanza los mayores niveles de actividad en mayo, para después mantener una presencia más reducida hasta el inicio del otoño, Erodius carinatus aparece con un tipo de ciclo bastante diferenciado de los anteriores, ya que limita su actividad a los meses primaverales, en los que llega al alcanzar unos niveles poblacionales muy elevados.

Por lo que respecta a las preferencias espaciales, en la tabla I se expresa el número medio de capturas por tampa en cada uno de los territorios definidos en el apartado anterior para el conjunto del año.

Destacan en primer lugar la relativamente escasa utilización de la zona $\mathrm{C}$ por parte de las cuatro especies. La zona A, por el contrario, aparece como la más frecuentada por tres de las especies: Erodius carinatus, Pimelia modesta y Pachychila frioli, mientras que Tentyria elongata muestra una mayor presencia en el territorio B. Debe señalarse, sin embargo, que ninguno de los territorios es excluido completamente por ninguna de las especies, y que en particular la zona $B$ registra un elevado porcentaje de las capturas totales de los coleópteros con preferencias por $\mathrm{A}$ y, de la misma manera, un gran número de Tentyria es capturado en la zona A.

Se han propuesto numerosos índices estadísticos para la cuantificación del estado agregativo de las poblaciones biológicas. Así, la varianza relativa $\left(\mathrm{s}^{2} / \overline{\mathrm{x}}\right)$,

\section{TABLA I}

CAPTURAS MEDIAS POR TRAMPA EN CADA TERRITORIO

\begin{tabular}{|c|c|c|c|}
\hline & A & B & $\mathrm{C}$ \\
\hline T. elongata ................... & 1,14 & 2,42 & 0,80 \\
\hline E. carinatus ................. & 11,27 & 7,60 & 2,14 \\
\hline P. frioli ...................... & 2,76 & 1,42 & 0,52 \\
\hline P. modesta .................... & 2,40 & 0,08 & 0,12 \\
\hline
\end{tabular}



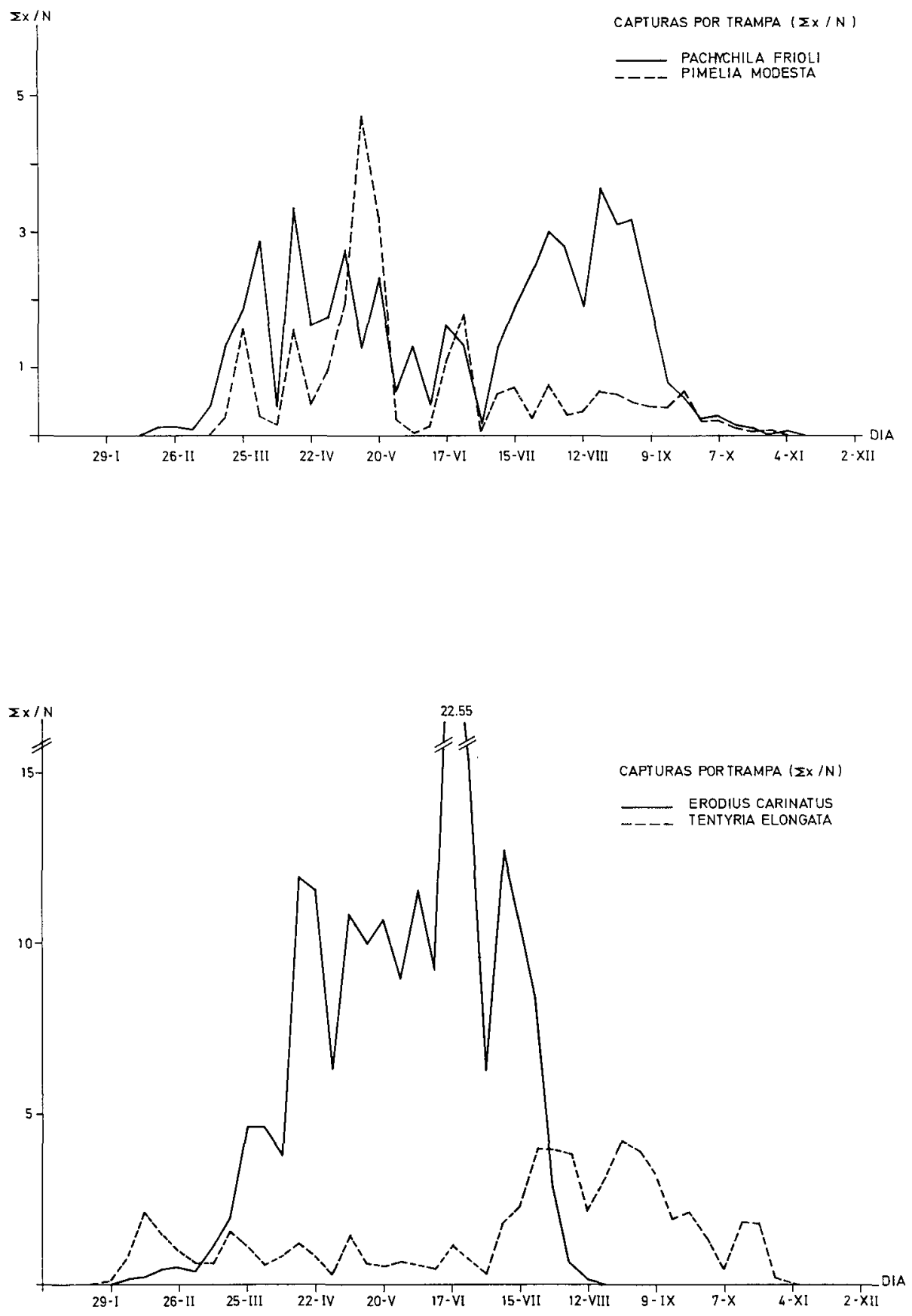

Fig. 3.-Periodo de actividad de cada especie, expresado en número de ejemplares capturados semanalmente por trampa. 
el índice de Lefkovich $\left(\mathrm{I}_{\alpha}\right)$, el «Mean crowding» (委), el de Taylor (b), etc. ( Vid. SOUTHWOOD, 1966; TAYLOR, 1984). De ellos, el que parecía más indicado para nuestro tipo de muestreo era el de Morisita $\left(\mathrm{I}_{\delta}\right)$, ya que aparenta ser, al menos hasta cierto punto, el menos influido por el tamaño muestral (Vid., no obstante, TAYLOR, 1984).

El índice de Morisita tiene la forma:

$$
I_{\delta}=\frac{\sum_{i=1}^{n} n_{i}\left(n_{i}-1\right) N}{n(n-1)}
$$

donde $\mathrm{N}=\mathrm{n} .{ }^{\circ}$ muestras, $\mathrm{N} \mathrm{i}=\mathrm{n} .{ }^{\circ}$ individuos en muestra $\mathrm{i}, \mathrm{n}=$ total de individuos en todas las muestras.

En la gráfica 4 aparece la variación de los $I_{\delta}$ de cada especie a lo largo del período de actividad. No se calcularon los índices de agregación de aquellos muestreos en que las capturas fueron excesivamente bajas.

Según estos resultados, Erodius carinatus parece mostrar a grandes rasgos unos mayores índices de agregación al principio de la temporada, que alcanzan su máximo en mayo para después mantenerse relativamente bajos hasta el cese de la actividad. Tentyria elongata, por su parte, inicia la actividad con unos bajos niveles de agregación, que se elevan fuertemente en mayo para luego mantenerse bajos hasta el inicio del otoño, en que registran un nuevo aumento. Pachychila frioli parece comportarse de manera similar, con máximos también en mayo y octubre. En el caso de Pimelia modesta, la escasez de capturas impide extraer ideas claras al respecto, aunque parecen insinuarse dos máximos relativos, uno en mayo y otro en junio.

TABLA II

LOCALIZACIÓN DE LAS AGREGACIONES DE CADA ESPECIE EN LOS PERÍODOS DE MAYOR $I_{\delta}$

\begin{tabular}{|c|c|c|c|c|c|c|c|}
\hline \multicolumn{2}{|c|}{$T . e}$. & \multicolumn{2}{c|}{ E. c. } & \multicolumn{2}{c|}{$P . f}$. & \multicolumn{2}{c|}{$P . m}$. \\
\hline $25-\mathrm{III}$ & $\mathrm{B}$ & $1-\mathrm{IV}$ & $\mathrm{A}$ & $29-\mathrm{IV}$ & $\mathrm{A}$ & $22-\mathrm{IV}$ & $\mathrm{A}$ \\
\hline $6-\mathrm{V}$ & $\mathrm{A}$ & $22-\mathrm{IV}$ & $\mathrm{A}$ & $20-\mathrm{V}$ & $\mathrm{A}$ & $13-\mathrm{V}$ & $\mathrm{A}$ \\
\hline $27-\mathrm{V}$ & $\mathrm{B}$ & $13-\mathrm{V}$ & $\mathrm{A}$ & $17-\mathrm{VI}$ & $\mathrm{A}$ & $17 \mathrm{VI}$ & $\mathrm{A}$ \\
\hline $24-\mathrm{VI}$ & $\mathrm{B}, \mathrm{B}$ & $3-\mathrm{VI}$ & $\mathrm{A}$ & $5-\mathrm{VIII}$ & $\mathrm{A}$ & $15-\mathrm{VII}$ & $\mathrm{A}$ \\
\hline $5-\mathrm{VIII}$ & $\mathrm{B}, \mathrm{C}$ & $17-\mathrm{VI}$ & $\mathrm{A}$ & $2-\mathrm{IX}$ & $\mathrm{A}$ & $29-\mathrm{VII}$ & $\mathrm{A}$ \\
\hline $30-\mathrm{IX}$ & $\mathrm{B}$ & $1-\mathrm{VII}$ & $\mathrm{A}$ & - & - & $23-\mathrm{IX}$ & $\mathrm{A}$ \\
\hline
\end{tabular}



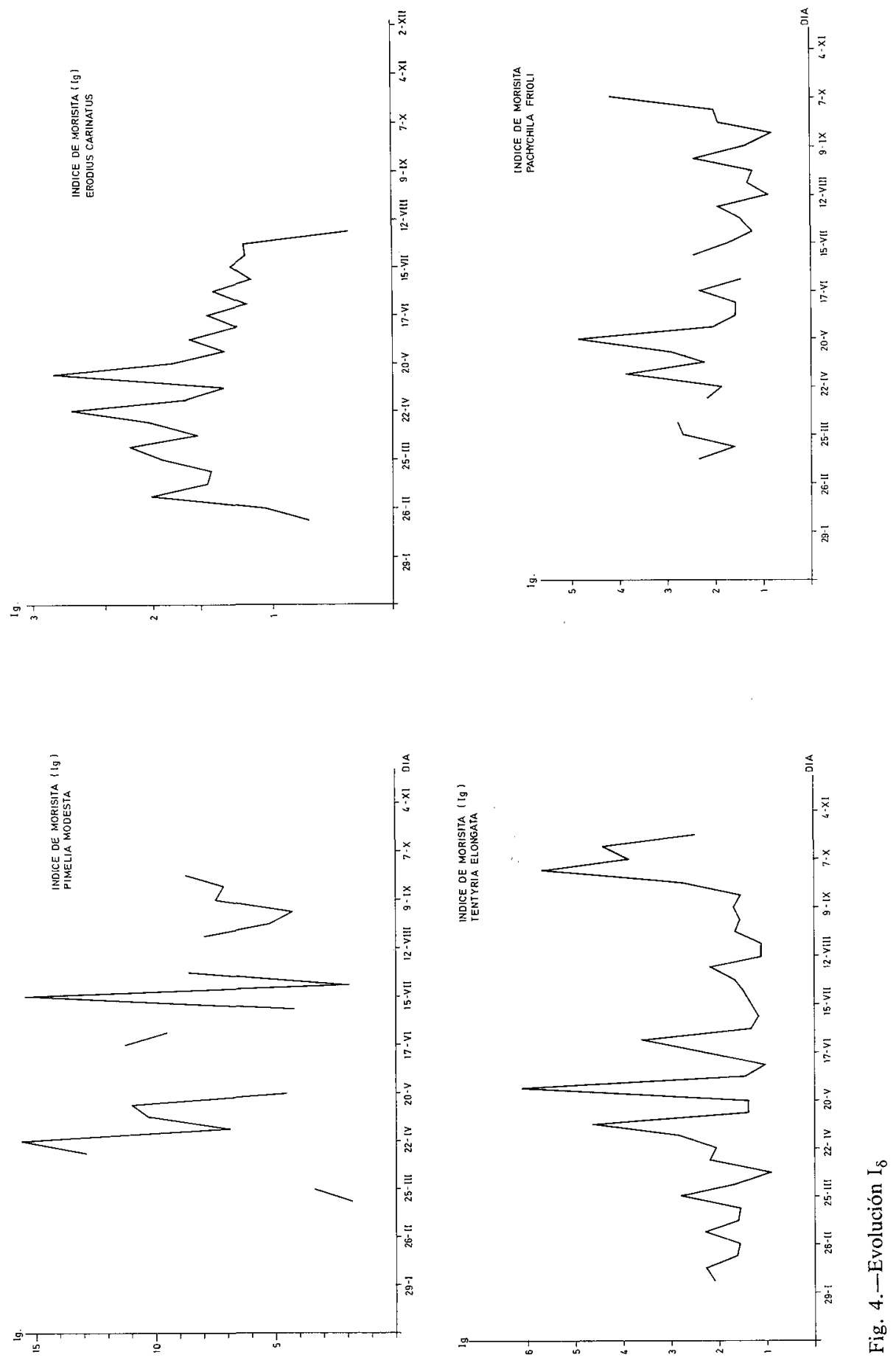


\section{DISCUSIÓN}

\section{A) Ciclos de actividad}

Contrariamente a lo que sucede con otros grupos de artrópodos apigeos como los Carabidae o Scarabaeidae, los ciclos vitales de los Tenebrionidae han recibido una atención relativamente escasa por parte de los investigadores. Anque algunas especies de Tenebrionidae de las zonas subdesérticas de Norteamérica y Sudáfrica han sido estudiadas al respecto con cierto detalle (p. ej., WISE, 1981 a y b; RICKARD \& HAVERFIELD, 1965; CALKINS \& KIRK, 1973; LOUW, 1985, 1986; WHICKER \& TRACY, 1987; PARMENTER \& McMAHON, 1982; RICHMAN \& al., 1982; MARSHALL, 1985), no se puede decir lo mismo de los representantes paleárticos de la familia. Los únicos datos que poseemos de los períodos de actividad de la mayoría de los taxones paleárticos son las fechas de captura que figuran en los especímenes museísticos. De algunas especies tenemos estudios ecológicos que proporcionan datos más o menos precisos de sus fenologías, mientras que en muy pocos casos se han llevado a cabo investigaciones con cierta profundidad. Ello, desde luego, impide realizar análisis comparativos y extraer ideas globales acerca de los patrones generales y líneas de variación de los modelos fenológicos de la familia, tal como se viene realizando en los Carabidae, por ejemplo (PAARMAN, 1979).

En la península Ibérica, el único estudio detallado de este tipo realizado en taxocenosis de Tenebrionidae, el de DE LOS SANTOS (1983), nos proporciona, sin embargo, un primer ensayo de caracterización fenológica de la familia. Distingue este autor dos grandes modalidades de ciclos de actividad: el de las especies de ciclo largo, prolongado desde marzo hasta octubre, y el de las especies de ciclo corto, normalmente limitado a los meses primaverales. Dentro del primer tipo, a su vez, cabe diferenciar entre poblaciones que presentan dos máximos de actividad, uno en primavera y otro a finales del verano, separados por un periodo de actividad estival muy reducida o nula, y poblaciones que alcanzan su máximo alrededor de mayo para después mantener una actividad menor hasta el inicio del otoño.

El modelo fenológico con período de actividad largo y bimodal parece ser bastante común entre los Tenebrionidae tanto paleárticos como de otras partes del mundo (Vid. RICHMAN \& al., 1982; MARCUZZI, 1981). Se trata del tipo de ciclo presentado en la comunidad de El Saladar por Pachychila frioli y $T$. elongata, aunque el pico primaveral de esta última sea relativamente temprano y poco marcado. Es interesante señalar que otros Tentyriini de los que poseen datos al respecto muestran también, con sólo algunas diferencias de detalle, este modelo fenológico. Así, Tentyria platyceps, de Andalucía Occidental (DE LOS SANTOS, 1983) y T. laevigata y $T$. grossa del sur de Italia (MARCUZZI, 1981). Los datos de BODENHEIMER (1934) sobre las especies palestinas $T$. herculanea, $T$. laticollis y $T$. discicollis, aunque poco precisos, también parecen indicar que dichas especies también presentan un período de actividad largo, desde primavera a otoño.

Pimelia modesta, por su parte, se ajusta bien a la modalidad de ciclo largo con máximo en mayo, al igual que la italiana $P$. rugulosa (MARCUZZI, 1981). Sin embargo, otras especies del género, como $P$. costata, de la Andalucía Occidental (DE LOS SANTOS, 1983) y $P$, bipunctata, de las dunas del sur de Francia (BRUN, 1970, 1975) pertenecen al grupo bimodal. Reviste particu- 
lar importancia la diferencia entre $P$. modesta y $P$. bipunctata, ya que viene considerándoselas como especies muy cercanas filogenéticamente (KWIETON, 1977). La muerte de las poblaciones primaverales de $P$. bipunctata durante el verano, con emergencia de una nueva generación al inicio del otoño, a pesar de las temperaturas estivales bastante benignas de la Camarga francesa en comparación con las de la costa alicantina, resulta difícil de explicar desde el punto de vista adaptativo si se admite que los ciclos de esta especie y de $P$. modes$t a$, por razón del parentesco filogenético, han de derivar de un antecesor común. De todas formas, la escasez de capturas de $P$. modesta durante esta campaña nos obliga a ser cautos al respecto.

Erodius carinatus es la única especie de nuestra taxocenosis que presenta un período de actividad corto y restringido a los meses primaverales. Cabe destacar que la única especie de esta tribu de la que conocemos con detalle su fenología Erodius goryi (DE LOS SANTOS, 1983), también muestra este tipo de ciclo. Igualmente, los datos parciales de BODENHEIMER (1934) parecen señalar este modelo de actividad estacional para las especies palestinas Erodius gibbus y E. 4-lineatus.

\section{B) Distribución espacial}

Aunque en buena parte de los estudios ecológicos realizados en comunidades de coleópteros terrestres se proporcionan datos sobre los tipos de microhábitat preferidos por las distintas especies, en pocos de ellos se tiene en cuenta el hecho de que la distribución espacial de las mismas es, de hecho, dinámica y varía a lo largo del período de actividad. Exposiciones detalladas de estos modelos espaciales, con cuantificación de niveles de agregación y las variaciones temporales de los mismos, pueden encontrarse en algunos trabajos referentes a Carabidae (GRUM, 1973; DEN BOER, 1979). En el caso de los Tenebrionidae, únicamente DE LOS SANTOS (1982) analiza estos aspectos en dos comunidades de la Andalucía Occidental. Sin embargo, en estos trabajos no se aborda apenas las posibles relaciones entre la ubicación de los aglomerados de coleópteros y las características del territorio.

Las peculiaridades espaciales de los ecosistemas dunares como el de la Platja del Saladar que hacen posible una fácil caracterización de los diferentes biotopos, nos han permitido comprobar, tal como se ha expuesto anteriormente, que los agregados de una especie concreta tienden a formarse siempre en un mismo tipo de territorio. Es importante señalar que, tal como puede comprobarse comparando las gráficas 1,2 y 3, no existe una relación evidente entre los niveles poblacionales y los valores de $\mathrm{I}_{\delta}$ lo cual descarta que haya una influencia directa de la densidad de actividad sobre tales índices. Cabe suponer, por tanto, que en ciertos momentos de su vida estos coleópteros «buscan» activamente aquellos territorios que les son, por una u otra razón, más favorables. De hecho, parece ser que estos comportamientos están en relación con los estados fisiológicos de los insectos, ya que algunos estudios han mostrado que los mayores índices de agregación se alcanzan durante los períodos de vitelogénesis (KREHAN, 1970; HURKA, 1975; DE LOS SANTOS, 1983). Evidentemente, ello ha de deberse a una intensificación de la actividad trófica en estos períodos de intenso anabolismo y la consiguiente acumulación de individuos en las zonas de mayor disponibilidad de alimentos. 
En especies ambulatorias como los coleópteros terrestres de superficie, los individuos, durante su búsqueda de alimento, pueden atravesar biotopos diversos, aunque no todos ellos les sean ecológicamente favorables (BAARS, 1979). No obstante, es obvio que su comportamiento será distinto en zonas ventajosas por su riqueza en alimento $u$ otras razones y en zonas que sean desfavorables para el animal. Los trabajos realizados hasta ahora muestran claramente que en las primeras el movimiento es más lento, con una alta frecuencia de giros y con distancias absolutas recorridas menores. En las zonas desfavorables o indiferentes el movimiento es más rápido, con menor frecuencia de giros, o incluso con un cierto mantenimiento de la dirección durante largos períodos de tiempo, y con distancias absolutas recorridas mayores (MOLS, 1979; BAARS, 1979). Ello, lógicamente, ha de traducirse en una mayor probabilidad de que un individuo caiga en una trampa situada en una zona favorable $\mathrm{y}$, por tanto, en un número de capturas más elevado en dichas trampas.

Existen, sin embargo, períodos en que las especies animales adquieren una actividad de tipo migrador o dispersivo y en los que la actividad trófica disminuye. Tal comportamiento es de especial importancia en el caso de especies que habitan biotopos discontinuos o inestables, ya que de esta manera colonizan territorios desocupados y se produce un cierto intercambio genético entre las distintas poblaciones (SOUTHWOOD, 1981). Resulta evidente que durante tales períodos ha de registrarse una presencia relativamente elevada de la especie en hábitat a los que, de hecho, no se encuentra ligada ecológicamente. Un análisis de las preferencias de hábitat realizado sin tener en cuenta estos aspectos, por tanto, podría conducirnos a una interpretación errónea de las verdaderas preferencias espaciales de dicha especie.

El estudio de las variaciones de los índices de agregación puede ser de gran utilidad en este sentido, ya que es de esperar que durante las fases dispersivas el muestreo refleje unos estados agregativos relativamente bajos, mientras que en los períodos de explotación activa del territorio tales índices han de ser mayores.

Así, pues, la localización de las trampas que registren un mayor número de capturas en períodos de elevado $\mathrm{I}_{\delta}$ puede indicarnos con cierta precisión el tipo de biotopo al cual una especie se halla más ligada. El que los agregados se sitúen casi siempre en un mismo biotopo inclina a suponer que en la formación de dichos agregados las características del medio desempeñan un papel de importancia y, por tanto, no pueden explicarse simplemente como resultado de fenómenos de atracción entre individuos por razones sexuales o de otro tipo (LUFF, 1986).

En el caso de los Tenebrionidae de El Saladar, las diferencias interespecíficas en cuanto a las preferencias de microbiotopo no pueden venir dadas únicamente por distintas preferencias alimentarias, dada la similitud de dietas entre estas especies ampliamente omnívoras, como es normal en la familia. Seguramente deba pensarse en distintas adecuaciones a las características del sustrato. Fijémonos en que las tres especies más ligadas a las zonas de $\mathrm{Am}$ mophilion, esto es, a las zonas de arenas menos consolidadas, poseen estructuras morfológicas claramente indicativas de hábitos fosoriales, como el ensanchamiento en forma de pala de las protibias de Erodius carinatus y Pachychila frioli, o la fuerte dilatación de los espolones metatibiales de Pimelia modesta. Estas especies excavan con gran habilidad en la arena y en ella se 
refugian durante los períodos de inactividad. Por el contrario, Tentyria elongata, que ocupa los territorios con cierto grado de fijación del sustrato, carece de estructuras morfológicas adaptadas a la excavación y, de acuerdo con ello, no presenta hábitos fosoriales, tal como hemos podido comprobar tanto en condiciones naturales como en cautividad. Normalmente, esta especie se refugia bajo piedras o troncos, o entre la hojarasca, enterrándose sólo someramente.

MEDVEDEV (1966) distinguía dos síndromes morfológicos generales entre los miembros de la familia Tenebrionidae. Por un lado, el de aquellas especies propias de terrenos consolidados, provistas de patas relativamente largas, bien adaptadas a la ambulación, y que tendían a refugiarse en grietas del terreno, madrigueras de animales o bajo piedras. Por otro lado, el de las especies que viven sobre sustratos no consolidados, como las dunas costeras o del desierto, que muestran diversas modificaciones en sus extremidades que les habilitan para excavar y refugiarse en el sustrato. A este último grupo pertenecen Erodius carinatus, Pimelia modesta y Pachychila frioli, mientras que Tentyria elongata corresponde al prototipo ambulatorial de Medvedev. Así, pues, la preferencia de $T$. elongata por las zonas de arenas con cierto grado de fijación, rasgo que la segrega del resto de la taxocenosis, es bastante significativa. Sin embargo, debe destacarse que, de todas formas, $T$. elongata no aparece nunca fuera de la estricta área dunar. Otra especie del género, $T$. laevis Sol., la sustituye en los terrenos áridos de la región. Pero es interesante mencionar que $T$. laevis, especie de amplia ecología que se extiende desde las sierras de Cazorla y Segura hasta las zonas litorales de Alicante y Murcia (ESPAÑOL, 1960), infeuda asimismo los arenales del interior de la provincia de Alicante (Sauleda, 1977), lo cual puede hacernos suponer que su exclusión de los arenales costeros se debe tan sólo a la presencia en los mismos de la T. elongata. En la playa de El Saler, de Valencia, donde no existe $T$. elongata ni ninguna otra Tentyria sabulícola estricta, la especie eurioica $T$. peiroleri Sol., que habita todo tipo de terrenos secos en una amplia zona del E. ibérico, y que muy probablemente deba sinonimizarse con T. laevis (ESPAÑOL, 1960), ocupa también las dunas costeras, tal vez con preferencias espaciales del tipo de las mostradas por $T$. elongata en El Saladar. Al respecto, conviene señalar que en la Italia meridional especies de muy amplia ecología como Tentyria italica Sol. y T. grossa Bess. habitan también los arenales costeros (MARCUZZI, 1982).

Todos estos aspectos tienen gran importancia en el estudio de la evolución del género Tentyria, ya que plantean el problema de cuáles son los mecanismos adaptativos que permiten al grupo sabulícola, que parece representar una rama filogenética real (ESPAÑOL, 1952, 1960) correspondiente al primer grupo taxonómico en que REITTER (1900) dividió el género, la exclusión de las dunas costeras de las especies del grupo generalista, a pesar de su relativa inadecuación al medio arenoso.

Son necesarios, de todas formas, más estudios detallados sobre los modelos de ocupación del espacio de las especies de Tenebrionidae para poder intentar la formulación de ideas generales al respecto. Pensamos que sólo mediante un mejor conocimiento de los patrones de distribución espacial, así como de los ciclos biológicos de estos coleópteros podremos alcanzar una buena comprensión no únicamente de su ecología, sino también de ciertos problemas evolutivos, y que por tanto tal conocimiento sería de enorme utilidad para la solución de los numerosos problemas taxonómicos que tiene planteados esta 
compleja familia. Las notables aportaciones que estudios de esta naturaleza han realizado al conocimiento relativamente profundo que actualmente tenemos de grupos como los Carabidae y los Scarabaeidae, son buena prueba de ello.

\section{AGRADECIMIENTOS}

Queremos expresar aquí nuestro agradecimiento al doctor Frances Español por su ayuda en la sistemática de los Tenebrionidae y su estímulo a la hora de iniciar este trabajo. Igualmente al doctor Antonio de los Santos, nos prestó su ayuda desde un principio y ha hecho interesantes sugerencias sobre algunos aspectos ecológicos. Debemos dejar constancia también de nuestra gratitud a los servicios forestales de la Conselleria d'Agricultura de la Comunitat Valenciana en Alicante, y en especial al ingeniero Don Juan Giner, por las facilidades de todo tipo que nos han prestado para la realización de nuestro trabajo. 


\section{BIBLIOGRAFÍA}

ADIS, J. 1989: Problems of interpreting Arthropod Sampling with Pitfall Traps. Zool. Anz. Jena 202: $177-184$.

BAARS, M. A. 1979: Patterns of Movement of Radioactive Carabid Beetles. Oecologia (Berlin) 44: $12-140$.

BODENHEIMER, F. S. 1934: Studies on the ecology of Palestinian Coleoptera: II Seasonal and diurnal appearnce and activity. Bull. Soc. Entomol. Egypte 18: 211-241.

BRUN, G. 1970: Cycle biologique de Pimelia bipunctata (Col. Tenebrionidae) dans les dunes du littoral provençal et languedocien. Ann. Soc. Ent. France (N. S.) 6 (3): 655-671.

- 1975: Recherches sur l'écologie de Pimelia bipunctata (Col. Tenebrionidae) des dunes du littoral de la Camargue. Bull. Ecol. 6 (2): 99-116.

CALKINS, C. O. \& V. M. KIRK. 1973: Distribution and Movement of Adult False Wireworm in a Wheat Field. Ann. Entomol. Soc. America 66 (3): 527-32.

DEN BOER, P. J. 1979: Populations of Carabid Beetles and Individual Behaviour. General Aspects. Misc. Papers Landbouw. Wabeningen 8: 14-49.

DESENDER, K. \& J. P. MAELFAIT. 1986: Pitfall Trapping within enclosures: a Method for Estimating the Relationship between the Abundances of Coexisting Carabid Species. Hol. Ecol. 9: 245-250.

DOYEN, J. T.\& W. F. TSCHINKEL. 1974: Population Size, Microgeographic Distribution and Habitat Separation in Some Tenebrionid Beetles. Ann. Entomol. Soc. Am. 67 (4): 617-626.

ESPAÑOL, F. 1952: Los Tenebriónidos terrícolas del Pirineo catalán. Pirineos 24: 21-251.

- 1960: Avance al estudio de los Tentyria ibéricos. Eos 36: 403-412.

GREENSLADE, P. J. M. 1964: Pitfall trapping as a method for studying populations of Carabidae. J. Animal Ecol. 33: 301-310.

GRUM, L. 1973: Patterns of Carabus arcensis, distribution within different habitats. Bull. Acad. Pol. Sci. 21: 229-233.

HURKA, K. 1975: Species abundance and organization. Ecology 40: 404-16.

KREHAN, I. 1970: The control of annual periodicity and diapause in overwintering larvae and adults of the genus Pterostichus Oecologia (Berlin) 6: 58-105.

KWIETON, E. 1977: Esquisse phylogénetique dugenre Pimelia F. Acta Entomol. Mus. Nat. Praga 39: $59-89$.

LUFF. M. L. 1982: Population dynamics of Carabidae. Ann. Appl. Biol. 101: 164-170.

- 1986: Aggregation of some Carabidae in Pitfall Traps. In: DN Boer \& al.: Carabid Beetles. Fisches, Stuttaart-New York.

LOUW, S. 1983: The diversity and Daily and Seasonal Activity of the ground-livind Tenebrionidae in the Sothern Namib and Kalahari Ecosystems. Cimbebasia 7 (3): 36-56.

- 1986: Periodicity in and ecological equivalence between the ground-livin Tenebrionidae in the Southern Namib and Kalahari Ecosystems with notes on phylogenetic relationships. Navors. Nas. Mus. Bloemfontein (12): 301-324.

MARCUZZI. 1981: Aspetti ecologici della Tenebrionidofauna del Mediterraneo. Ecol. Mediterran. 7 (2): 103-118.

- 1982: Ecologia dei Tenebrionidi (Insecta, Coleoptera) dell'ecosistema litorale sabbioso della Puglia e della Provincia di Matera (Italia) Quaderni sulla Struttura delle Zoocenosi Terrestri. 3: Ambienti mediterranei, I Le coste sabbiose. Roma.

MARSHALL, L. D. 1985: Seasonal Patterns of Reproduction in two Species of Desert Beetles (Coleoptera, Tenebrionidae). Fla. Entomol. 68 (4): 621-627.

MEDVEDEV, H. S. 1965: Adaptations of leg structure in desert darkling beetles (Coleoptera, Tenebrionidae). Entomol. Rev. 44: 473-48.

MOLS, P. J. M. 1979: Motivation and walking behaviour in the Carabid beetle Pterostichus coerulescens $\mathrm{L}$. at different densities and distributions of the prey. A preliminary report. Misc. Papers Landbouw. Wageningen 18: 185-198.

PAARMANN, W. 1979: Ideas about the evolution of the various annual reproduction rhythms in Carabid beetles of the different climatic zones. Misc. Paper. Landbouw. Wageningen 18: 119-132.

PARMENTER, R. R. \& MACMAHON. 1984: Factors influencing the distribution and abundance of ground-dwelling beetles in a shrub-steppe ecosystem: the role of shrub architecture. $\mathrm{Pe}$ dobiologia 26: 21-34.

RICHMAN, D. B. \& al. 1982: Seasonal activity of Tenebrionidae beetles in Nwe Mexico mesquite dunes. Southwest. Nat. 27 (3): 30-308. 
RICKARD, W. H. 1970: The distribution of ground-dwelling beetles in relation to vegetation, season and topography in the Rattlesnake Hills, Southeastern Washington. Northwest Science 44 (2): 107-113.

RICKARD, W. H. \& L. E. HAVERFIELD. 1965. A pitfall trapping survey of darkling beetles in desert steppe vegetation. Ecology 46 (6): 873-87.

SAULEDA, N. 1977: Contribución al conocimiento de la fauna halófila y psammófila de la provincia de Alicante (Coleoptera y Heteroptera), su relación con la vegetación y creación y explotación de un banco de datos faunísticos. Tesis Doctoral. Universidad de Valencia.

SEVA, E. 1982: Taxocenosis de lacértidos en un arenal costero alicantino. Tesis Doctoral. Universidad de Alicante.

SOUTHWOOD, T. R. E. 1966: Ecological Methods. Chapman \& Hall. London.

- 1981: Ecological aspects of insect migration. In: Aidley (Ed.): Animal Migration. Cambridge University Press.

TAYLOR, L. R. 1984: Assessing and interpreting the spatial distribution of Insect populations. Ann. Rev. Entomol. 29: 321-57.

WHICKER, A. D. 1987: Tenebrionid beetles in the shortgrass prairie: daily and seasonal patterns of activity and temperature. Ecol. Entomol. 12: 97-108.

WISE, S. H. 1981a: A removal experiment with darkling beetles: lack of evidence for interspecific competition. Ecology 62: 727-738.

- 1981b: Seasonal and Yearly patterns in the densities of darkling beetles (Coleoptera, Tenebrionidae) in a Montane commnunity. Environ. Entomol. 10 (3): 350-358. 